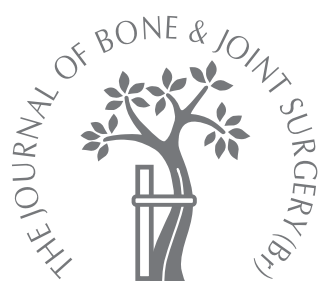

\title{
Coccygectomy: an effective treatment option for chronic coccydynia
}

\author{
RETROSPECTIVE RESULTS IN 41 CONSECUTIVE PATIENTS
}

A. M. Trollegaard, N. S. Aarby, S. Hellberg

From Holbaek Hospital, Holbaek, Denmark
A. M. Trollegaard, MD, Staff Surgeon

N. S. Aarby, MD, Resident

I S. Hellberg, MD, Orthopaedic

Surgeon

Department of Orthopaedic

Surgery

Holbaek Hospital,

Smedelundsgade 60, 4300

Holbaek, Denmark.

Correspondence should be sent to $\operatorname{Dr}$ A. M. Trollegaard; e-mail: akulstrup@gmail.com

(C)2010 British Editorial Society of Bone and Joint Surgery doi:10.1302/0301-620X.92B2 $23030 \$ 2.00$

$J$ Bone Joint Surg [Br] 2010;92-B:242-5.

Received 1 July 2009;

Accepted after revision 16

September 2009

\begin{abstract}
Between 1993 and 2008, 41 patients underwent total coccygectomy for coccydynia which had failed to respond to six months of conservative management. Of these, 40 patients were available for clinical review and 39 completed a questionnaire giving their evaluation of the effect of the operation. Excellent or good results were obtained in 33 of the 41 patients, comprising 18 of the 21 patients with coccydynia due to trauma, five of the eight patients with symptoms following childbirth and ten of 12 idiopathic onset. In eight patients the results were moderate or poor, although none described worse pain after the operation. The only post-operative complication was superficial wound infection which occurred in five patients and which settled fully with antibiotic treatment. One patient required re-operation for excision of the distal cornua of the sacrum.

Total coccygectomy offered satisfactory relief of pain in the majority of patients regardless of the cause of their symptoms.
\end{abstract}

Coccydynia was first described by Simpson in 1859. ${ }^{1}$ Its aetiology remains relatively undefined. ${ }^{2,3}$ It may result from direct injury such as a fall or indirect trauma after parturition. Coccydynia temporally related to childbirth might be considered traumatic but some rare cases are due to infection, cysts or tumours. ${ }^{4}$ However, causation in many cases cannot be related directly to any objective, radiological or other pathological findings and these cases are classified as idiopathic in origin. Traumatic coccydynia is thought to occur more frequently than the idiopathic type. ${ }^{4,5} \mathrm{MR}$ scanning has failed to correlate coccydynia with coccygeal hypermobility. ${ }^{6}$ In a radiological study of the anatomy of the coccyx, four configurations were defined based on the increasing anterior curvature and subluxation of the sacrococcygeal joint. ${ }^{7}$ The authors related the severest configurations directly to the intensity of pain in patients with coccydynia and the subsequent benefit from coccygectomy. Patients with coccydynia usually describe a pulling or piercing pain when sitting or during defecation. ${ }^{4,8}$ The differential diagnosis includes other anorectal disorders, with differentiation helped by injecting local anaesthetic into the pericoccygeal area. ${ }^{9}$ Conservative management is successful in about $90 \%$ of patients ${ }^{10}$ using an assortment of treatments, including nonsteroidal anti-inflammatory drugs, hot baths, ring-shaped cushions, manual therapy, massage, injections of local anaesthetic with corticosteroid, radiotherapy and psychotherapy. ${ }^{4,9,11-13}$ However, for some patients these measures do not help and surgery may be the only recourse. ${ }^{2,14}$

It has been reported that patients with low back pain due to degenerative disease or a prolapsed intervertebral disc seem to experience a limited benefit from coccygectomy and so these disorders are often considered as a contraindication to operation. ${ }^{15,16}$ Improvement following coccygectomy is often only fully appreciated after several months and patients should be warned accordingly. ${ }^{17}$ There are few published reports on chronic coccydynia treated with total coccygectomy. ${ }^{8,12,18,19}$ We report the results of 41 patients who underwent coccygectomy during a 16-year period.

\section{Patients and Methods}

We identified 41 consecutive patients with a mean age of 39.1 years (16 to 77 ), of whom 39 were women, with coccydynia for at least six months, a relevant history and pain on palpation of the coccyx, who underwent total coccygectomy in our department between 1993 and 2008. The result of their treatment was analysed retrospectively. In 21 patients the aetiology was trauma, in eight patients the pain arose post-partum and in 12 cases, the aetiology was unknown and was classified as idiopathic. Coccygectomy was indicated in 
these patients because of chronic coccygodynia which remained unresponsive to conservative treatment provided by their general practitioner and/or a referring hospital in the six months that preceeded referral to us. To our knowledge, no patient was suffering from a psychiatric disorder. Many of the patients were offered local infiltration around the posterior coccygeal periosteum and associated soft tissue. Where given, the infiltration consisted of $9 \mathrm{ml}$ of lidocaine $2 \%$ combined with $20 \mathrm{mg}$ triamcinolone. If this local infiltration reduced the coccydynia, this was considered support for the recommendation of surgery. All patients had pre-operative radiographs (one lateral and one anteroposterior) taken of the sacrococcygeal area.

Surgery was undertaken by the same surgeon $(\mathrm{SH})$ in all patients. With the patient in the prone position a $5 \mathrm{~cm}$ to $6 \mathrm{~cm}$ vertical midline incision was made directly over the coccyx. Dissection was carried down to the bone, followed by release of the anococcygeal ligaments using Mayo scissors. The distal part of the coccyx could then be elevated using a towel clip. The scissors were advanced under the anterior aspect of the coccyx, thereby protecting the rectum when separating the coccyx from the sacrum with an osteotome. A total coccygectomy was performed. Attention was paid to meticulous haemostasis and bone wax applied to the distal bleeding sacrum. All patients received intravenous cefuroxime per-operatively. After the operation the patients were allowed to mobilise freely.

The success of the procedure was judged by the patient's report of significant relief of coccygeal pain without postoperative complications when seen for outpatient review between three and four months later. In 2008 a questionnaire was sent to all 41 patients, of whom 39 responded, at a mean of 82.8 months (5 to 187 ) post-operatively. They were asked to assess their long-term pain relief on one of five levels (pain free, significant pain relief, some pain relief, unchanged pain level, worsened pain), to score their current pain level on a visual analogue scale (VAS) with 0 being no pain and 10 worst pain imaginable, to describe any improvement in their general quality of life (a validated classification was not used) and to state whether they would recommend coccygectomy to a patient with a similar level of pre-operative pain as themselves.

An excellent result was defined as being pain free or with significantly decreased pain, with a maximum VAS of 2 , and a major improvement in activities of daily living, including sitting and standing. A good result was significantly decreased pain but pain still present with a VAS of 3 or 4; a moderate result was some decrease of pain but pain still present with a VAS $>4$. Both of these categories exhibited improvement in the activities of daily living, including sitting and standing. A poor result was no decrease of pain and no or marginal improvement in the activities of daily living.

\section{Results}

With the exception of the trauma itself, there was no clear difference between the patients in the traumatic and in the idiopathic groups. The radiological findings did not appear to have a strong relationship to either the history or the level of pain (Table I). The mean duration of hospital admission was 1.6 days (one to six) with all 41 patients mobilised bearing full weight within the first post-operative day.

All patients but one were available for a routine outpatient review with a mean follow-up period of 4.9 months (1 to 42). Only three patients were reviewed clinically beyond seven months, at 18, 24 and 42 months; they had idiopathic, post-partum and post-traumatic coccydynia, respectively. Only the patient with an idiopathic origin had a poor outcome. There were two deaths of patients in the period since their operation, leaving 39 patients who were sent the questionnaire, all of whom completed it. The patient who did not undergo clinical review completed the questionnaire at 182 months and reported a moderate improvement. The two deceased patients had reported themselves to be very satisfied at their last outpatient review, at three and 42 months, respectively. For the purpose of the evaluation they were classified as having excellent results.

Of those who completed the questionnaire, 31 had excellent or good results, five had a moderate improvement and three had a poor result. In Table II the results for all patients are summarised according to the aetiology of the coccydynia. The mean VAS for the patients completing the questionnaire was 2.6 (0 to 10). A further operation 15 months after the primary coccygectomy was required in one patient who had post-partum coccydynia; the two distal sacral cornua were removed. She was slim and had pain at that location which was improved after their excision. She was moderately improved with a VAS of 6 in the questionnaire at 44 months. Of the three patients with poor results, none had any systemic disease nor ongoing insurance claims. One was intolerant to opiates and could take only nonsteroidal anti-inflammatory drugs. She suffered from severe headaches and was referred to physiotherapy. Another patient had lumbar pain which pre-dated the coccygectomy. No further action was taken as her last outpatient clinic attendance occurred only two months postoperatively, but she requested no further review when 40 months later she recorded a VAS of 7 in the questionnaire. The final patient who described a poor outcome in the questionnaire had been very satisfied at the outpatient review, but described severe coccygeal pain with a daily VAS of 7 in the questionnaire nine months after operation. He had undergone operative treatment for a lumbar disc herniation five years previously and still suffered from some lumbar pain at the time of operation. None of these patients had returned to the outpatients clinic for a further follow-up visit. All other patients experienced improvement in their condition. All patients with excellent and good results for pain relief also described a significant improvement in the activities of daily living, including sitting and standing, but these improvements were not quantifiable. No patients suffered any neurovascular or bowel 
Table I. Radiological findings

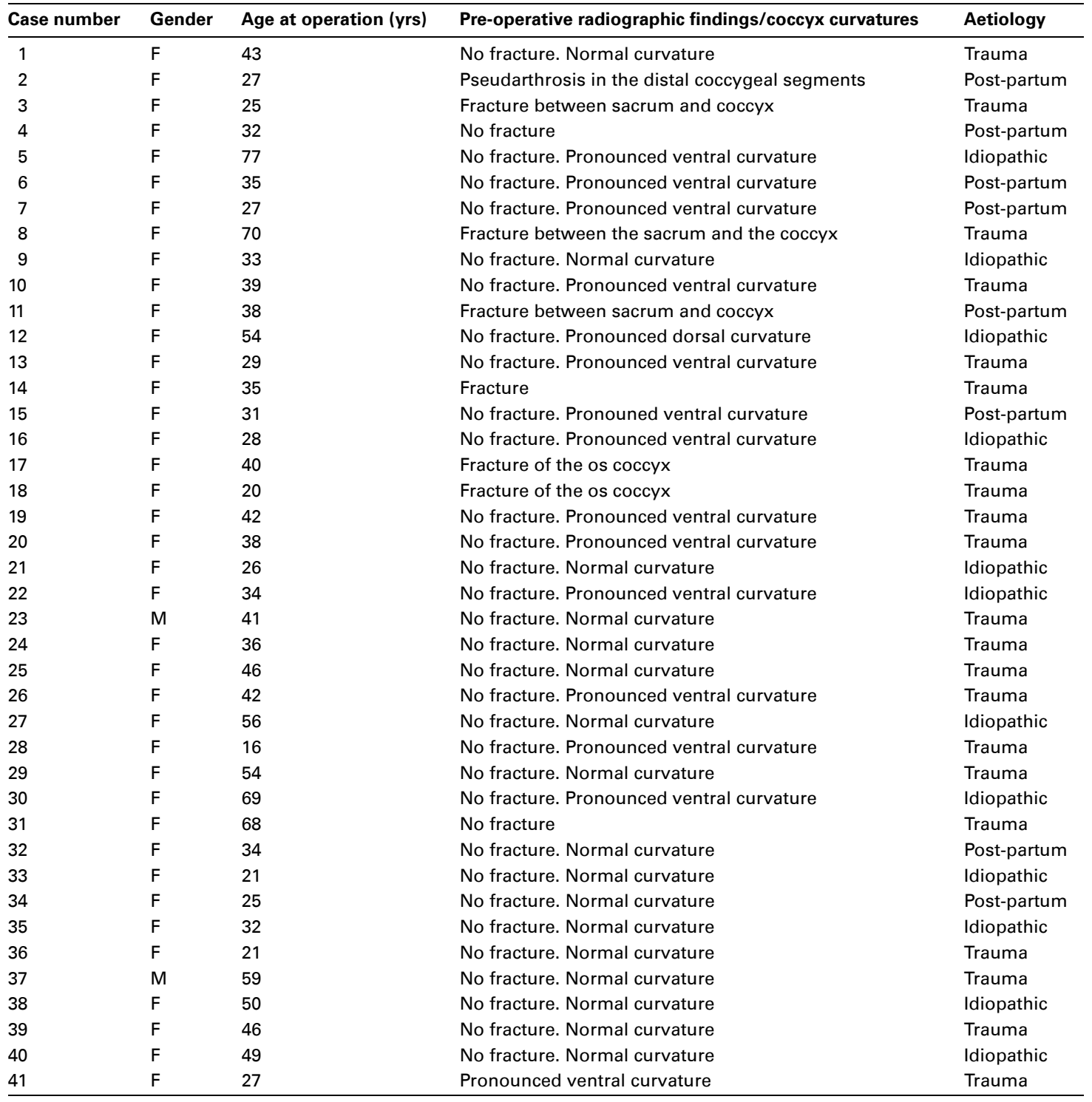

Table II. Patient outcome according to the aetiology of the coccydynia

\begin{tabular}{lcclc}
\hline & All patients (\%) & Traumatic coccydynia (\%) & Post-partum (\%) & Idiopathic (\%) \\
\hline Excellent & $25(61)$ & $13(62)$ & $4(50)$ & $8(67)$ \\
Good & $8(20)$ & $5(24)$ & $1(13)$ & $2(17)$ \\
Moderate & $5(12)$ & $2(10)$ & $2(24)$ & $1(8)$ \\
Poor & $3(7)$ & $1(4)$ & $1(13)$ & $1(8)$ \\
Total & $41(100)$ & $21(100)$ & $8(100)$ & $12(100)$ \\
\hline
\end{tabular}

injury, but five patients had a superficial wound infection that resolved with oral antibiotics and routine care. There were no deep infections.

In the group of 21 patients who had coccydynia of traumatic origin, 20 stated that they would recommend the procedure to a patient with similar pre-operative symptoms, as would 11 of the 12 patients in the idiopathic group.

\section{Discussion}

In this study, 18 of the 21 patients with traumatic coccydynia benefitted from coccygectomy, as did ten of the 12 patients in the idiopathic group. These numbers correspond fairly well with three other studies comparing traumatic and idiopathic coccydynia, with $75 \%, 88 \%$ and $76 \%$ success rates for traumatic coccydynia and 
$58 \%, 38 \%$ and $58 \%$ for those with idiopathic onset, respectively. ${ }^{5,15,18}$ Our findings correspond with reported success rates ranging from $71 \%$ to $100 \% .^{7,10,12,13,17,19-27}$ Infection and damage to the bowel ${ }^{28-30}$ have been reported as complications, but in our series only five patients had a superficial infection, all of which resolved with antibiotics alone. It is possible that if we had extended the antibiotic prophylaxis to 48 hours as recommended in one study, ${ }^{18}$ we might have reduced the incidence of infection.

Our study was limited by the lack of detailed information concerning the full extent of conservative treatment and compliance prior to referral to our care. It would have been helpful to have known the patients' activities of daily living and VAS for pain prior to surgery in order to assess more certainly the benefit derived from the coccygectomy.

The pre-operative radiological findings did not appear to have any relationship with symptoms or objective findings. The radiographs were reviewed by a radiologist and the surgeon $(\mathrm{SH})$ but were not graded according to any system of classification. Although this would have been interesting, it would not have changed the indications for surgery which we used. Patient selection depended on a failure to respond to conservative management which had been followed for at least six months and confirmation of the origin of the pain by temporary pain relief after local anaesthetic and corticosteroid infiltration. A lateral radiograph of the sacrococcygeal area was always obtained to define the shape of the coccyx and to avoid complications at operation. The literature is divided on whether total or partial coccygectomy should be undertaken. Hellberg and Strange-Vognsen ${ }^{17}$ found a higher incidence of surgical failure with partial coccygectomy. Wray and Templeton ${ }^{25}$ advised total coccygectomy, and Eng et $\mathrm{al}^{16}$ obtained $67 \%$ good results in 27 patients with only partial coccygectomy. In our study, all patients underwent total coccygectomy.

In our series of 41 carefully selected patients with coccygodynia who had undergone initial non-operative treatment for at least six months, ${ }^{7,17}$ total coccygectomy provided a satisfactory outcome in the majority of cases. The outcome was similar whether the symptoms were traumatically induced or idiopathic in origin.

No benefits in any form have been received or will be received from a commercial party related directly or indirectly to the subject of this article.

\section{References}

1. Simpson J. Clinical lectures on the diseases of women. Lecture XVII: coccygodynia and diseases and deformities of the coccyx. Medical Times Gazette 1859;40:1-7.

2. Johnson PH. Coccygodynia. J Ark Med Soc 1981;77:421-4.

3. Kothari S. Neuromodulatory approaches to chronic pelvic pain and coccygodynia. Acta Neurochir Suppl 2007;97:365-71.

4. Mlitz H, Jost W. Coccygodynia. J Dtsch Dermatol Ges 2007;5:252-4.

5. Pennekamp PH, Kraft CN, Stütz A, et al. Coccygectomy for coccygodynia: does pathogenesis matter? J Trauma 2005;59:1414-19.

6. Grassi R, Lombardi G, Reginelli A, et al. Coccygeal movement: assessment with dynamic MRI. Eur J Radiol 2007;61:473-9.

7. Postacchini F, Massobrio M. Idiopathic coccygodynia: analysis of fifty-one operative cases and a radiographic study of the normal coccyx. J Bone Joint Surg [Am] 1983;65-A:1116-24.

8. Doursounian L, Maigne J-Y, Faure F, Chatellier G. Coccygectomy for instability of the coccyx. Int Orthop 2004;28:176-9.

9. Fogel GR, Cunningham PY 3rd, Esses SI. Coccygodynia: evaluation and management. J Am Acad Orthop Surg 2004;12:49-54.

10. Capar B, Akpinar N, Kutluay E, Miejde S, Turan A. Coccygectomy in patients with coccydynia. Acta Orthop Traumatol Turc 2007;41:277-80 (in Turkish).

11. Patel R, Appanagari A, Whang P. Coccydynia. Curr Rev Musculoskelet Med 2008;:1:223-6.

12. Balain B, Eisenstein S, Alo GO, et al. Coccygectomy for coccygodynia: case series and review of literature. Spine 2006;31:414-20.

13. Feldbrin ZF, Singer M, Keynan 0, Rzetelny V, Hendel D. Coccygectomy for intractable coccygodynia. Isr Med Assoc J 2005;7:160-2.

14. De Andres J, Chaves S. Coccygodynia: a proposal for an algorithm for treatment. J Pain 2003;4:257-66.

15. Bayne O, Bateman JE, Cameron HU. The influence of etiology on the results of coccygectomy. Clin Orthop 1984;190:266-72.

16. Eng JB, Rymaszevski L, Jepson K. Coccygectomy. J R Coll Edinb 1988;33:202-3.

17. Hellberg S, Strange-Vognsen HH. Coccygodynia treated by resection of the coccyx. Acta Orthop Scand 1990;61:463-5.

18. Cebesoy O, Guclu B, Kose KC, et al. Coccygectomy for coccygodynia: do we really have to wait? Injury 2007;38:1183-8.

19. Sehirlioglu A, Ozturk C, Oguz E, et al. Coccygectomy in the surgical treatment of traumatic coccygodynia. Injury 2007;28:182-7.

20. Grosso NP, van Dam BE. Total coccygectomy for the relief of coccygodynia: a retrospective review. J Spinal Disord 1995;28:328-30.

21. Hodges SD, Eck JC, Humphreys SC. A treatment and outcomes analysis of patients with coccygodynia. Spine J 2004;4:138-40.

22. Karalezli K, Iltar S, Irgit K, et al. Coccygectomy in the treatment of coccygodynia. Acta Orthop Belg 2004;70:583-5.

23. Kim NH, Suk KS. Clinical and radiological differences between traumatic and idiopathic coccygodynia. Yonsei Med J 1999;40:215-20.

24. Wood K, Mehbod A. Operative treatment for coccygodynia. J Spin Disord Tech 2004; 17:511-15.

25. Wray AR, Templeton J. Coccygectomy: a review of thirty-seven cases. Ulster Med J 1982;51:121-4.

26. Zayer M. Coccygodynia. Ulster Med J 1996;65:58-60.

27. Bilgic S, Kurklu M, Yurttas $\mathbf{Y}$, et al. Coccygectomy with or without periosteal resection. Int Orthop 2009; Epub.

28. Garcia FJ, Franco JD, Marquez R, Martinez JA, Medina J. Posterior hernia of the rectum after coccygectomy. Eur J Surg 1988;164:793-4.

29. McClenahan JE, Fisher B. Herniation of the rectum following coccygectomy. Am J Surg 1951;82:288-9.

30. Zook NL, Zook EG. Repair of long-standing coccygeal hernia and open wound. Plast Reconstr Surg 1997:100:96-9. 